Noname manuscript No.

(will be inserted by the editor)

\title{
Transmission of kinematic information in dense granular systems: local and nonlocal network sensing
}

\author{
David M. Walker · Antoinette Tordesillas* · Amy \\ L. Rechenmacher
}

Received: date / Accepted: date

\begin{abstract}
We study how kinematic information propagates in plane strain compression tests for two granular samples, one comprising glass beads, the other is sand. Of interest are the structures of directed networks constructed from static linear relationships amongst grainscale kinematical measurements obtained using Digital Image Correlation (DIC). The exact form and kinematical information used in each linear relationship is selected using a data modelling algorithm which appeals to the information theory description length philosophy encapsulated by the principle of Occam's Razor. For both tests, we find that the observation sites with the most complicated relationships (i.e., those which require the $x-$ and $y$-coordinates of the displacements of both neighbouring and distant sites to best represent their own kinematics) are located in that region where the persistent shear band develops. The static linear relationships for these sites involve a length scale that is around $7-15$ times the mean particle diameter, consistent with the observed thickness of the shear band in each sample. Our findings corroborate earlier evidence from the extant literature that the kinematics inside shear bands are necessarily nonlocal and further highlights the crucial importance of incorporating shear band kinematics in constitutive modelling. We shed new insights not only for constitutive modelling but also in the use of sensors to detect motion in deforming granular systems: that sensors with local sensing and monitoring capabilities are sufficient for distilling information on kinematic transmission - except in the shear band where nonlocal information, or information from spatially distant sensors, is a necessity.
\end{abstract}

PACS 81.05.Rm

D. M. Walker

Department of Mathematics \& Statistics, University of Melbourne, Parkville VIC 3010 Australia

*A. Tordesillas (corresponding author)

Department of Mathematics \& Statistics, University of Melbourne, Parkville VIC 3010 Australia

Tel.: +61-3-83445550

Fax: +61-3-83444599

E-mail: atordesi@unimelb.edu.au

A. L. Rechenmacher

Department of Civil \& Environmental Engineering,

University of Southern California, Los Angeles CA 90089-2531 USA 
Keywords Digital image correlation; directed networks; granular media; kinematics; sensor networks

\section{Introduction}

A continuum constitutive model that can adequately describe the physics of granular media remains elusive. Continuum mechanics formulations for such discrete materials are built from kinematical relationships derived using two main approaches: (i) direct consideration of individual grain kinematics (e.g., grain motion in a confined buckling of a force chain [32, 33]), or, (ii) prescription of an assumed relationship among grain kinematics in a representative volume element (e.g., affine [5] or nonaffine [29, 21] deformation). If we know how the collections of grains move in a given granular assembly, then we can feed their displacements into formalisms which return information on the forces between grains and, in turn, the stresses and strains that develop in the representative continuum. In some respects, the kinematics provides the link from the discrete to the continuum. Here we demonstrate that directed complex networks, built from linear relationships among grain properties (i.e., their displacements), can be used to elucidate how properties of the discrete medium can be more effectively mapped to its continuum representation. By distinguishing the essential from the nonessential relationships in the system, this study may yield valuable clues towards constitutive models which are simple enough to be analytically tractable and yet robust enough to capture and predict observed behaviour.

To illustrate the key idea, suppose a continuum model does exist that can robustly represent and predict how a granular material behaves under an applied load. Depending on the relevant spatial scale of resolution, i.e., representative volume element, such a model should be capable of describing how collections of grains are moving in a particular region of the sample at a given time. Presumably a useful continuum model would take the form of a system of partial differential equations which may be approximated and decomposed to a system of ordinary differential equations. In either case, for practical computational purposes, the differential equations would be converted to a set of difference equations which would then be used to compute the displacement at mesh points given the displacements at other mesh points at the current and previous times. Furthermore, if we want to know the displacements at non-mesh points, then it is conceivable to approximate this as a function of the motions computed at nearby mesh points. Such a functional relationship may be static, i.e., only involve information at the current time. To simplify matters even further, we could assume the functional relationship is linear - that is, if we know the kinematics of the system at specific points in time, then we can predict the motion of all the other points in the sample using "static linear relationships" or SLR. Perhaps the best example of such linear relationships among grain kinematics is the affine or uniform strain assumption of continuum theory. The affine relationship, and the departure of observed granular deformation from this relationship (i.e., nonaffine deformation) has received significant attention in many areas of granular mechanics and physics, with recent studies including [21, 11, 13] from simulations to $[24,38]$ from experiments.

Before proceeding, it is useful to consider how information may be obtained for studies of SLR among various system properties, kinematics or otherwise, from either physical measurements or model predictions, e.g., discrete element simulations (DEM). Consider, for instance, the possibility of extracting information on properties of individual grains or clusters of grains of various mesoscopic sizes (e.g., kinematics, contact forces etc.) scattered in different parts of the sample, from a network or collection of smart sensors located inside 
the material - instead of using, say, a continuum model (differential equations) to predict such information. This sensor network would have the same material properties as any material grain or cluster in the sample. Thus, except for their unique capacity to record system properties (e.g., position, displacement, rotation, contact forces etc.) and transmit them to an observer, they are otherwise indistinguishable from any constituent grain or cluster of grains. Armed with these sensor measurements and a suite of static linear relationships, the observer can then approximate the motion or property of any grain or cluster in the material. In this study, we are interested in discovering the structure of such linear relationships from grain-scale measurements of displacements.

A twofold question of relevance to this study concerns how kinematical information propagates through the material: (i) what sites within the material require the most complicated relationships (i.e., nonlocal relations describing interaction with distant sites or sensors, as opposed to local relations which is that with immediate neighbours) to track rheological behaviour, and (ii) where in the material should the sensor network reside so that optimal approximations of grain motions at distant locations in the sample can be obtained. The answer to this second question has great practical import not just for basic research in geoengineering (e.g., constitutive modelling), but also for the applied area of wireless sensor networks for remote sensing and real-time monitoring of nonlocal phenomena (i.e., geomaterial failure such as landslides and earthquakes) in the geological and geophysical communities, e.g., [15, 22, 6, 41].

In this study, we attempt to partially answer both questions by introducing and demonstrating our techniques using data from experimental digital image correlation (DIC) measurements of grain-scale kinematics of assemblies of glass beads and a masonry-concrete sand mixture. We treat each grain-scale measurement site (here fixed in space) as a sensor, and derive static linear relationships between the sensors for a given loading history. Information theory is utilized to determine which set of sensors are the optimal or best selection of sensors to predict the behaviour of another sensor. This information can be conveniently summarized within a directed network. Of specific interest in this study are structure and properties of these directed networks and the insights they provide into the rheological behaviour of granular materials.

We take advantage of current, local-scale experimental techniques (DIC) that enable accurate, non-destructive measurement of deformation at this scale of interest (grain cluster scale) to deliver complete records of kinematics at all observation sites for the entire duration of the loading program $[25,1]$. The method of DIC yields overlapping grain-scale displacements, with consecutive measurement points about a grain apart. In this study, we consider grain-scale DIC measurements of two plane strain compression tests of disparate materials whose difference stems largely from differences in grain shape, i.e., an assembly of glass beads and a masonry-concrete sand mixture. As expressed in [4], rarely in a dense granular system will you see a single grain moving in a completely different manner from its neighbours (e.g., spinning uncontrollably, slipping or moving in a dramatically contrary manner to its neighbours). Due to frictional resistance and particle interlocking, a grain's rotation invariably entails the rotation of its immediate neighbours. Consequently, measuring movements of small clusters of grains across experimentally realizable strain intervals with DIC is a valid way of quantifying the essential kinematical activity, including that inside shear bands, as recently demonstrated in [25] and [1].

It is appropriate to make clear that the method of analysis we propose in this paper is not restricted to solely processing grain-scale DIC data. For example, recent advances in $\mu$-CT $\mathrm{X}$-ray technology warrant mention since measurements from these techniques can also be treated in a similar fashion. In contrast to the measurement sites of DIC the moving individ- 
ual grains would serve as the sensors, since $\mu$-CT x-ray technology now enable individual grain kinematics to be tracked across a deformation history through a method called IDtrack [3]. This gives the experimentalist the ability to return kinematic information in real sand tests akin to the information typically delivered from DEM simulations [9]. However, despite the successful progress of ID-track, it is not perfectly refined and individual grains can be "lost" to the tracking [3]. This causes difficulty for the methods we propose in this study, where a complete record of grain information is required for the analysis. The method of DIC does not suffer from this loss of tracking and as mentioned above DIC still delivers grain-scale kinematical information and so we use DIC data to introduce and develop our methods.

The remainder of this paper is organized as follows. In Sec. 2 we describe the experimental tests and DIC data collection. The computational methods proposed are described in Sec. 3 with results presented in Sec. 4. Finally, a summary of the techniques and further implications for constitutive theory are discussed in Sec. 5.

\section{Experiment \& Data}

We subject low porosity, prismatic-shaped specimens (approx. 140 by 40 by $80 \mathrm{~mm}$ ) of sand and glass beads to plane strain compression. We enforce plane strain conditions by rigid glass walls, which enable image-based capture of in-plane specimen deformation. The specimens sit on a translatable base which is kinematically free to slide only in the in-plane direction, thus allowing for unconstrained translation along an inclined shear band. A pliable and translucent latex membrane encases each specimen, which is saturated to enable measurement of volumetric strain by measuring pore water exchange into and out of the specimen. A large-diameter Plexiglas cell houses the specimen assembly. Pressurized silicon oil within the cell provides the medium for confining stress application. Axial deformation rates are small, about $0.05 \mathrm{~mm} / \mathrm{min}$., to avoid effects of pore water over-pressurization. Every $0.025 \%$ global axial strain, or every $45 \mathrm{sec}$ throughout shear, digital images are collected, as well as readings of macroscopic axial and out-of-plane forces and axial and in plane displacements.

The digital imaging technique used here (described below) requires the subject material to possess local material colour variations at the scale of interest. To achieve the required variation in our granular samples, we mixed together materials of different colour. The sand material is a $90 / 10 \%$ mixture by mass of sieved masonry $(M)$ and concrete (C) sands, herein called MC sand. Prior to mixing, we sieved each sand between the No. $16(1.18 \mathrm{~mm})$ and No. $30(0.6 \mathrm{~mm})$ sieves to create a fairly uniform grain size distribution. The median grain size (by mass) is $0.84 \mathrm{~mm}$. The glass bead material is a bi-disperse mixture of $1.0-\mathrm{mm}$ opaque orange ( $40 \%$ by mass) and $1.5-\mathrm{mm}(60 \%$ by mass) opaque purple, green and yellow beads. The median grain size is roughly $1.25 \mathrm{~mm}$. All specimens are prepared by raining dry material into a forming mould from a consistent height throughout filling: a filling height of about $12 \mathrm{~cm}$ consistently produced high bulk density specimens, which are prone to shear band formation. Typical macroscopic stress-strain data are given in Fig. 1 for the glass beads and sand specimens. The highlighted data points on the curve indicate image points referenced in the discussions below.

DIC is used to compute in-plane displacements across the out-of-plane specimen surface. DIC mathematically tracks pixel gray level value patterns manifested within small subsets of pixels (e.g., [30]). The subset sizes used here comprise about three to four grains across. As is typical in DIC, the subsets are overlapped to yield a spatially intense dis- 
placement mapping: here, our centre-to-centre subset spacing is equivalent to about a grain, yielding grain-scale displacement data point spacing. The software VIC-2D, by Correlated Solutions, Inc. is used to conduct the DIC analyses. We use the Zero Normalized Sum of Squared Differences (ZNSSD) matching criterion, cubic spline sub-pixel interpolation, and affine subset shape functions. Incremental DIC analyses are performed every $0.15 \%$ axial strain, representing about $4 \%$ gross shear strain across a shear band. For these small strain increments, and for the subset sizes used here, the assumption of affine subset deformation is fairly well maintained [25]. Local kinematic behaviour and nonaffine deformation, then, are assessed by evaluating the relative kinematics among adjacent DIC data points [7]. Figures 2 and 3 show the observed displacement fields at four strain increments corresponding to the image numbers shown in Fig. 1. A single persistent shear band develops in each sample, the measured thickness of which is around 7 mean grain diameters for the glass beads assembly and 9 mean grain diameters for the masonry-concrete sand. More detail about the testing and DIC techniques can be found in [25] and [1].

\section{Computations}

We adapt methods previously used to characterize dynamics of observed time series from noisy experimental observations. Specifically, we build static linear relationships (SLR) valid for all stages, or times $t$, in the loading history, from consideration of the time series of the $x$ - and the $y$-coordinates of the displacement field at every observation site in the sample, and for the entire loading history for which DIC is ideally suited to provide. The static linear relationships or SLR among grain-scale kinematics can then be used to predict an observation site's kinematics at a fixed time $t$ - using knowledge of other sites' kinematics at $t$. Thus the predictive capability of these static relationships is with respect to space only and not time. Furthermore the relationships are conceived from knowledge (i.e., time series) of grain-scale kinematics at many observations sites in the sample for all of its loading history. More general models incorporating time structure and causal relationships could be considered. One such class of models is Hidden Markov Models [12]. These models can be regarded as consisting of two parts: a state evolution model capturing the underlying dynamics and a measurement model describing how one projects the (hidden) states to the observations. It is a difficult and open question as to what constitutes, or is suitably representative of, the state of a granular system and so, in this study, we restrict ourselves to identifying dependences between instantaneous observations of a granular material throughout loading by reconstructing SLR's.

The technique used to construct the SLR for a given site at time $t$ is a modification of the reduced autoregressive (RAR) model fitting methods developed for detecting periodicity in (possibly) nonlinear data [28]. The RAR method involves the use of an information criterion, namely description length, to select which linear combination of past values of a time series, or lags, is best able to fit the current value of the time series without overfitting the data. The use of description length in this way has been extended to nonlinear combinations of past values of the time series with success [14]. The description length of a model of a time series can be thought of as the information required to reconstruct the original data. If one was to transmit the information needed to reconstruct the original data, then the description length is the compression gained from transmitting the information needed to build the SLR and transmitting its prediction errors — rather than simply transmitting the original data [27]. A 
formula for approximating the description length of a model takes the form

$$
\begin{aligned}
\text { description length } \approx & \text { (number of data) } \times \ldots \\
& \ln (\text { prediction errors })+\ldots \\
& (\text { penalty for number and } \ldots \\
& \text { accuracy of model parameters })
\end{aligned}
$$

The approach by Judd \& Mees [14], inspired by the work of Rissanen [26], provides a formula for description length which involves solving a nonlinear optimization problem for the parameter accuracy. Space limits prohibit a full explanation here, but to solidify the concept, we will introduce the Schwarz Information Criterion (SIC), which is a simpler information criterion to the description length used in [14] but can still match its success and description length is asymptotic to SIC in the limit of large number of data. In time series modelling applications the difference in formula for description length and SIC often do not lead to significant differences in "optimal" model structure for small number of data - the case here with 30 time points for each site, however, DIC is capable of delivering a finer temporal resolution. Indeed for the studies here it is possible to extract up to 10 times the number of data. Typically SIC models contain more terms but the essential core features of these larger models encompass the smaller "optimal" description length built models [18]. For a static linear relationship with $k$ terms (or parameters), the SIC formula is

$$
\operatorname{SIC}(k)=N \ln \left(\mathbf{e}^{T} \mathbf{e} / N\right)+k \ln N
$$

where $N$ is the length of the time series to be fitted and $\mathbf{e}$ are the fitting errors.

In [28, 17, 31], RAR models are reconstructed using a scalar time series. In our case, however, we reconstruct, for each coordinate of an observation site, a predictive SLR. This SLR is a linear combination of the coordinate time series of the observed displacement at a subset of other sites. Specifically, the SLR for $u_{i}(t)$ and $v_{i}(t)$, which predicts the $x-$ and $y$-coordinates, respectively, of the observed displacement at site $i$ at time $t$, is

$$
\begin{aligned}
& u_{i}(t)=\lambda_{0}+\sum_{c \in S_{u x}(i)} \alpha_{c} u_{c}(t)+\sum_{c \in S_{u y}(i)} \beta_{c} v_{c}(t)+e_{u} \\
& v_{i}(t)=\hat{\lambda}_{0}+\sum_{c \in S_{v x}(i)} \gamma_{c} u_{c}(t)+\sum_{c \in S_{v y}(i)} \delta_{c} v_{c}(t)+e_{v}
\end{aligned}
$$

where the $\lambda_{0}, \hat{\lambda}_{0}, \alpha_{c}, \beta_{c}, \gamma_{c}$ and $\delta_{c}$ are parameters estimated using least squares to minimize $e_{u}$ and $e_{v}$. The sets $S_{u x}(i), S_{u y}(i), S_{v x}(i)$ and $S_{v y}(i)$ are the subsets of observation sites, not including site $i$ itself, which have been selected as having the richest kinematic information throughout the time history to predict the evolving displacements observed at site $i$, with respect to the description length information criterion in (1). For example, $S_{u x}(i)$ is the set of sites whose $x$-coordinate displacement information is required to predict the $x$-coordinate displacement information of site $i$. These subsets are determined in an iterative growing and culling algorithm established in [14].

Briefly, the growing and culling algorithm results from a sensitivity analysis in Lagrangian theory [42] to select candidate sites to add to and to remove from the subsets. Equation (2) can be rewritten as a matrix equation $r=X p+e$ where $r$ is the response variable (i.e., the observed displacements of site $i$ through time), $p$ are the parameters, $X$ is a design matrix whose columns correspond to the observed displacements of the currently selected sites in the subsets. The quantity $e$ represents the fitting errors for the given parameters. The information (displacements) of sites not currently in the subsets also form their own design matrix, say $V$. We grow the model subsets by adding a new sites information (a column of $V$ ) by selecting the one which has the maximum value of $\left|V^{T} e\right|$. Lagrangian theory 
says that adding this site to form an extended $X$ gives the maximum payoff given the current makeup of the subsets and those available for selection [14]. The culling component of the selection algorithm again appeals to Lagrangian theory and dual variables by selecting the site (or column of $X$ ) which has the smallest absolute value of fitted parameter as removal of this information provides the least damage to the fit [14]. After the addition and deletion of a sites information the SIC (equation (1)) of the resulting model is calculated and compared to the current "best" model. Recall, by "best" we mean the one with lowest SIC seen so far. For a model of fixed size $k$ once iterative growing and culling fails to reduce SIC further we increase the model size to $k+1$ and repeat the growing and culling steps. Early on, increasing model size will continue to lower SIC because the models errors $e$ reduce. Eventually, however, the payoff in reducing $e$ is outweighed by the increasing $k$ and SIC will start to rise defining a minimum of SIC for some $k$. Once we have a well-defined minimum, or $k+i$ for $i=1, \ldots, K$ for a given $K$ has failed to reduce SIC further we stop and consider the model with lowest SIC we have found so far as the "best" model. Of course, there is no guarantee that we have found the optimal model but the heuristic outlined above has proven successful in capturing the essential underlying behaviour in other applications [14, 28, 18]. A different or stricter description length criterion to SIC often leads to smaller models (selected subsets) than SIC, however, the core features of the underlying process is also captured by SIC [18].

The parameters $\lambda_{0}$ and $\hat{\lambda}_{0}$ are also considered part of the subset for selection, and so may or may not be present in the model of $u_{i}(t)$ and $v_{i}(t)$ for site $i$. The membership of each model (i.e., the subsets $S_{u x}(i), S_{u y}(i), S_{v x}(i)$ and $\left.S_{v y}(i)\right)$ can be usefully summarized in a directed network in a manner similar to and inspired by [17, 31]. Since SIC models capture the core features of a process the resulting core structure of the corresponding directed networks are also captured. Any extra terms in SIC models above "optimal" models constructed using the more complicated description length typically manifest as branches and leafs around the network core [17].

In $[17,31]$, weighted directed networks embodying the time structure of scalar time series were constructed. Those networks were developed by first assigning a node to each scalar value of the time series, and then adding weighted directed links if earlier values of the time series (nodes) appeared in the best RAR model. The links were assigned weights based on a transformation of the coefficients of the fitted RAR models. Our approach using the above predictive SLR (equation (2)) and multivariate time series differs in a novel way which we now explain. In particular, in our new approach, network nodes represent the spatial location of measurement sites and the type of information each site records.

The nodes of our network represent each measurement site coordinate (i.e., each of the multivariate kinematic time series). Thus for $M$ measurement sites, we have $2 M$ directed network nodes since we build the SLR for both displacements $u_{i}$ and $v_{i}$. We connect a directed link in to node $i$ from other nodes $j$, if and only if site $j$ is selected in the relationship for $i$ using equations (1) and (2). If the kinematic information of site $j$ is selected by (1) in (2), then clearly node $j$ has an out link. We ignore the $\lambda_{0}$ term, regardless of it being included in the best relationships as it does not represent a site directly. In future work, it might be interesting to create nodes representing this constant term. Furthermore, at this stage of development, we do not include the transformation of the coefficients to obtain weights for the links as in $[17,31]$. A weighted graph would allow different aspects of the network to be interrogated. For example, in $[17,31]$ a weighted representation allowed a minimal weighted spanning tree to be extracted which bore important implications for causal relationships and model redundancy. We are unsure at this stage how such a minimal spanning tree and its properties translates to the spatial status of nodes and so restrict the scope here to a study of the less complicated unweighted network topology of SLR's. We reserve the addition of the 
weights to a future investigation which will also consider more general feedback predictive relationships than the SLR described in (2).

Of most interest then are the in-degree of a site's kinematic SLR, or the number of other measurement locations deemed best to predict its kinematic behaviour. The out-degree of a site's SLR is also key and is the number of times a given site's kinematical information appears in the SLR of other sites. Similarly informative are the types of information that are used in the SLR of a given site. That is, for a given site $i$, what information from the other sites $j$ is selected to best predict its displacement coordinates $u_{i}$ and $v_{i}$ ? Would the prediction of $u_{i}$, the $x$-coordinate of the displacement at site $i$, need only the $x$-coordinate of the displacements of the other sites $j$ (i.e., non-empty $S_{u x}(i)$ but $\left.S_{u y}(i)=\emptyset\right)$, or only the $y$-coordinate $\left(S_{u x}(i)=\emptyset, S_{u y}(i) \neq \emptyset\right)$, or both $\left(S_{u x}(i) \neq \emptyset, S_{u y}(i) \neq \emptyset\right)$ ? Similar questions apply for predicting $v_{i}$, the $y$-coordinate of the displacement at site $i$.

\section{Results}

One may expect that each SLR, being a linear model of a profoundly nonlinear process, would perform poorly especially since each SLR tries to fit the observed behaviour across the entire loading history. To the contrary, the SLR (training) fits of a site's observed behaviour using the information of a subset of other sites' data are reasonably good. We demonstrate this in Figs. 5 and 7, wherein we show the observed data and the quality of the SLR (training) fit for four sites in each sample corresponding to the locations indicated in Figs. 4 and 6 respectively. In both samples, we selected three sites (Sites $1-3$ ) in the region where the persistent shear band develops and where the static linear relationships have relatively complex structures (i.e., $S_{u x}(i) \neq \emptyset, S_{u y}(i) \neq \emptyset$ ). We chose the fourth site (Site 4$)$ to lie outside the shear band, where the SLR structure was less complex. The left hand plots in Figs. 5 and 7 show the $x$-coordinate information and the right hand plots show the $y$-coordinate information. The Sites 1-4 correspond to each row in these figures. The SLR fits (symbols in the plots) to the measurement site training data (solid line in the plots) produce fitting errors with zero mean, and a standard deviation that is at least an order of magnitude smaller than the range of the observed displacement data. We also note that the worst training fits are for those sites situated in the eventual shear band region and occur at strains where each material fails. This is best exemplified in the MC sand sample at Site 3 for $y$-coordinate information (Fig. 7, third row right).

Of central interest to this study is the structure of the static linear kinematic relationships uncovered for the glass beads and MC sand samples. This structure is the same for the entire loading history: the fitted parameters are constant and information from the entire loading history is used to fit the model parameters in equations (1) and (2). In what follows, we will examine this structure from multiple perspectives. We begin with the standard and highly informative first step in complex network analysis which is the representation of a complex network through its adjacency matrix. This matrix is typically a square matrix whose $i j^{\text {th }}$ element is equal to 1 if a link exist between nodes $i$ and $j$, otherwise it is 0 . In an undirected network, the $i j^{\text {th }}$ entry equals the $j i^{\text {th }}$ entry: the adjacency matrix is symmetric. For a directed network, this is not always the case. If we follow the convention whereby a nonzero $i j^{\text {th }}$ entry represents a link from node $j$ to node $i$, or the link is an in-link to $i$, it does not necessarily mean that the out-link from node $i$ to node $j$ exists, i.e, a nonzero $j i^{t h}$ entry. In Fig. 8, the adjacency matrices for the directed networks for both samples are presented such that the first half of the rows represent the $x$-coordinate information of the displacements while the remaining half of the rows represent the $y$-coordinate of the 
displacements. In both cases, a prominent diagonal can be observed highlighting that, for the majority of the sample, the best linear relationships for $u_{i}$ and $v_{i}$ are those which use the same type of kinematical information at nearby sites, i.e., $u_{i}\left(v_{i}\right)$ is expressed in terms of the $x$-coordinate ( $y$-coordinate) of displacement at nearby sites. However, it can also be seen from the off-diagonal entries that some sites' SLR require both nonlocal as well as mixed information, where nonlocal means information from nearby as well as distant sites are needed and mixed means that information from both $x$ - and $y$-coordinates are needed.

In a directed network, a fundamental summary of the network lies in the in-degree and out-degree distributions. Using our convention, the empirical in-degree distribution (outdegree distribution) corresponds to the row sum (column sum) of the adjacency matrix entries. We show these empirical in-degree and out-degree distributions for both samples in Fig. 9 plotted on a log-log scale. In both systems, the typical static linear relationships only involves a few terms as evident in the low in-degree (typically, no higher than $3-5$ ): in other words, only a few other sites contribute to a given site's displacement. The average out-degree is only marginally higher compared to the average in-degree but the empirical out-degree distributions have longer tails than their in-degree counterpart (Fig. 9): this implies that a typical site contributes to the displacement of only a few other sites in the sample, with some sites (those in shear bands) contributing moderately more so than others. Therefore, overall, we find no evidence of "minority influence" with respect to the transmission of information on kinematics: that is, there are not specific sites whose kinematic history are selected in the majority of the observation sites' SLR. The log-log scale plots of the empirical degree distributions in Fig. 9 are suggestive of a power law relationship. Indeed, for each distribution one can find a best fit parameter for the slope of this straight line, however, such a fit does not pass a rigorous statistical Kolmogorov-Smirnov test of significance [8]. We are thus unable to say with any certainty that the form of the in-degree and out-degree distributions are best explained by a power law.

We next examined the spatial length scales associated with the static linear relationships with respect to the average Euclidean distance from a site to its selected SLR sensors, as expressed in terms of the mean particle diameter $\bar{D}$. We compute each length scale according to the type of the selected SLR for transmission of kinematic information, categorized as follows: Type $1-u=L(u), v=L(v)$; Type $2-u=L(u, v), v=L(v)$; Type $3-u=L(u), v=L(u, v)$; and Type $4-u=L(u, v), v=L(u, v)$. Category 1 implies that the reconstructed static linear relations to predict the $u(v)$ displacement only use $u(v)$ displacement information, while the latter three categories of $2-4$ all use mixed information. We find that the SLR in these latter mixed categories involve an average length scale in the range $(7-12) \bar{D}$ for the glass beads sample and $(9-15) \bar{D}$ for the MC sand (see, Fig. 10). The length scales for the mixed SLR are consistent with the observed thickness of the shear band for both samples: as mentioned in Sec. 2, the measured thickness was 7 and 9 mean grain diameters for the glass beads and MC sand specimens, respectively. That the SLR predicts an upper bound thickness likely results from the nature of the DIC data upon which the SLR are based. Recall that DIC captures the average subset displacements. As discussed in [25], this leads to DIC data reflecting an over-estimation of shear band thickness. While the experimental measurements reported in [25] qualitatively account for this overestimation, applying the quantitative procedures discussed therein to compensate for the DIC bias results in a shear band thickness of around 5 mean grain diameters for the glass beads and 7 mean grain diameters for the MC sand. These latter values correlate with actual grain displacements analyzed from the digital images themselves. The larger thickness for the MC sand is likely a consequence of heightened grain interlocking among the irregularly shaped grains $[25,1]$. 
Finally, we present the structure of the kinematic transmission according to the four aforementioned categories of reconstructed static linear relationships - now mapped to their respective sample domain. In Figs. 11 and 12 representing the glass beads and MC sand samples respectively, the symbols marking the observation sites of the samples correspond to the structure, or type, of the SLR. The dotted sites represent SLR which belong to the one category (Type 1) that is not mixed, i.e. $u=L(u), v=L(v)$. The filled squares represent SLR of the form $u=L(u, v), v=L(v)$, the cross marked sites are of the form $u=L(u), v=L(u, v)$ and the unfilled circle sites consist of SLR of the form $u=L(u, v), v=L(u, v)$. Sites $1-$ 3 , selected in the earlier analysis in Figs. 4 and 6 belong to these latter categories. What is intriguing is that despite the tests involving vastly different materials with respect to particle shape, those sites requiring more complicated kinematical relationships reside in the shear band for both systems (see the displacement fields for the final strain increment in Figs. 2 and 3 as a guide on the location of the strain localization region). The region in which sites have SLR's with mixed and nonlocal information is more sharply demarcated in the MC sand test, i.e., Fig. 12. This distinction in kinematic transmission between the nodes inside versus outside of the shear band being more sharply portrayed in the MC sand, compared to the assembly of idealized spherical glass beads, can be explained by the greater interlocking among constituent grains in the MC sand. A qualitative analysis of the glass beads specimen corroborates this: constituent glass beads more readily exhibit intergranular slip, both within the shear band and at the shear band boundary. In particular, these continual grain motions across the band boundaries in the glass beads specimen (i.e., grains constantly going into and out of the shear band) result in constant fluctuations in the observed band thickness. We note that the structure of these simple SLR's shown in Figs. 11 and 12 have captured the very essence of the considerable "noisy" characteristics of granular material behaviour. That is, our use of an information criterion (i.e., SIC) to "filter" out nonessential relationships has resulted in SLR structures which reflect the complexity inherent to the phenomena we are seeking to understand.

The above trends are in distinct contrast to our findings elsewhere for force transmission [40]: there we discovered that certain grain sensor force histories were always favoured in the static linear relationships of other grain sensors. These favoured grains or sensors were found to reside in the shear band. Thus, with respect to description length, the force histories of grains inside the shear band were of use in the SLR of grains outside of the shear band. Thus, the prediction of forces on a grain involved nonlocal as well as local information. This makes intuitive sense if one considers force transmission in a granular material. Consider, for example, the forces on a constituent grain of a force chain, the average length of which typically lies in the range $5-20$ particles [16]. The forces this grain experiences is much the same as the other grains in the chain, over the course of that force chain's history (i.e., all are above global average and in the same general direction of the maximum or most compressive principal stress). Hence, the forces on any one of the member grains predict the forces of any other grain in the chain, no matter how distant the grains are from each other. When the force chain fails, however, the richest information is contained in the immediate vicinity of the chain. Hence, from the perspective of force transmission - local as well as nonlocal information is necessary for reliable predictions. On the other hand, as discussed above, with respect to the measure of description length, local information is generally more important and sufficient in the transmission of kinematical information. Thus, sensors with local sensing and monitoring capabilities are sufficient for distilling information on grain kinematics - except in the shear band where nonlocal information is still a necessity. 


\section{Conclusion}

A directed network has been used to characterize how kinematic information propagates in two granular assemblies comprising of different materials, i.e., glass beads and mixed masonry-concrete sand. Static linear relationships among kinematical information, obtained at different observational sites (or sensor network) in the sample using DIC measurements, has been summarized as a directed complex network. The in-degree and out-degree distributions give information on how much kinematic information from other sites are needed for good predictions. Only a few other sites, typically less than 5 (approximately $0.1 \%$ of all sample sites), govern a given site's displacement and vice versa.

We also found that the sites in the shear band hold the most useful information, as well as requiring the most information from all the other sites in the sample. In particular, we showed that those sites having the most complicated static linear relationships - i.e., those requiring information on both $x$ - and $y$-coordinates of both nearby and distant sites to predict its own $x$ - or $y$-displacement component - existed in areas of the material where the persistent shear band eventually formed. Kinematic transmission in these areas of the sample exhibited a spatial length scale that is in the range $7-15$ times the mean particle diameter, consistent with the observed shear band thickness.

The regions outside a fully developed band are essentially in rigid-body motion, whereas experiments and simulations have shown that the shear band region exhibits a range of highly interdependent processes spanning multiple spatial scales, including: relative sliding and relative rolling of particles [2], continual formation and collapse of force chains [32, 20, $35]$ and contact cycles [36], interlacing contractant and dilatant regions [10, 23] and vortices [25, 34]. Moreover, force balance must apply across the boundaries of the shear band, thus these dynamical processes also depend on the regions outside (e.g. when the shear band undergoes a slip event, the regions outside unload elastically). Features of this interaction between the shear band and the regions outside can be found in [39] where relationships between grains with respect to their force history showed that groups of grains predominately in the shear band shared similar response behaviour with some grains residing in the rigid-body-like areas.

Our findings provide partial answers to the two questions we sought to answer (i) shear band sites require the most complicated relationships and (ii) the majority of sensors should ideally be placed in the likely shear band region, e.g., a fault gouge, but some sensors placed outside of this area are also required. Overall our results corroborate previous findings that shear band kinematics is nonlocal and encompass those grains with the richest kinematic history (e.g., see [32, 33, 21, 3, 4, 1, 23, 19] and references cited therein).

Our results also suggest that the development of robust continuum models of granular systems depend crucially on capturing the kinematics of those grains in shear bands. Accordingly, ongoing studies are focussed on the fine details of the interdependencies among grain motions with respect to key mesoscopic events inside shear bands (e.g., birth-death evolution of force chains $[19,37]$ and vortices $[21,1])$. These are designed to elucidate the root causes of nonlocality and mixing in kinematical information transfer in discrete media and, in turn, the most essential kinematical relationships needed to construct robust yet tractable continuum models.

Acknowledgements We thank Dr. John Peters for valuable insights and useful comments. This work was partially supported by the US Army Research Office (W911NF-11-1-0175), the Australian Research Council (DP0986876 and DP120104759) and the Melbourne Energy Institute (AT, DMW). ALR is supported by USA National Science Foundation (NSF) grant CMMI-0748284. 


\section{References}

1. Abedi S, Rechenmacher AL, Orlando AD (2012) Vortex formation and dissolution in sheared sands. Granular Matter 14:695-705

2. Alonso-Marroquin F, Vardoulakis I, Herrmann HJ, Weatherley D, Mora P (2006) Effect of rolling on dissipation in fault gouges. Physical Review E 74:031,306

3. Andò E, Hall SA, Viggiani G, Desrues J, Bésuelle P (2012) Grain scale experimental investigation of localised deformation in sand: a discrete particle tracking approach. Acta Geotechnica 7(1):1-13

4. Bardet JP, Proubet J (1991) A numerical investigation of the structure of persistent shear bands in granular media. Geotechnique 41:599-613

5. Bathurst RJ, Rothenburg L (1988) Micromechanical aspects of isotropic granular assemblies with linear contact interactions. Journal of Applied Mechanics 55:17-23

6. Bernstein J, Miller R, Kelley W, Ward P (1999) Low-noise MEMS vibration sensor for geophysical applications. Journal of Microelectromechanical Systems 8:433-438

7. Chupin O, Rechenmacher AL, Abedi S (2012) Finite strain analysis of non-uniform deformations in shear bands in sand. International Journal of Numerical and Analytical Methods in Geomechanics 36:1651-1666

8. Clauset A, Shalizi CR, Newman MEJ (2009) Power-law distributions in empirical data. SIAM Review 51:661-703, companion toolbox http://www.santafe.edu/ aaronc/powerlaws/

9. Cundall P (1989) Numerical experiments on localization in frictional materials. Ingenieur-Archiv 59(2):148 - 159

10. Desrues J, Viggiani G (2004) Strain localization in sand: an overview of the experimental results obtained in Grenoble using stereophotogrammetry. International Journal for Numerical and Analytical Methods in Geomechanics 28:279-321

11. Ellenbroek WG, Zeravcic Z, van Saarloos W, van Hecke M (2009) Non-affine response: jammed packings vs. spring networks. Europhysics Letters 87:34,004

12. Elliot RJ, Aggoun L, Moore JB (2010) Hidden Markov Models: Estimation and Control, isbn-10: 1441928413 edn. Springer

13. Griffa M, Daub EG, Guyer RA, Johnson PA, Marone C, Carmeliet J (2011) Vibrationinduced slip in sheared granular layers and the micromechanics of dynamics earthquake triggering. Europhysics Letters 96:14,001

14. Judd K, Mees A (1995) On selecting nonlinear models for nonlinear time series. Physica D 82:426-444

15. Liu J, Zhao F, Cheung P, Guibas L (2004) Apply geometric duality to energy-efficient non-local phenomenon awareness using sensor networks. IEEE Wireless Communications 11:62-68

16. Muthuswamy M, Tordesillas A (2006) How do interparticle contact friction, packing density and degree of polydispersity affect force propagation in particulate assemblies? Journal of Statistical Mechanics: Theory and Experiment 06:P09,003

17. Nakamura T, Tanizawa T (2012) Networks with time structure from time series. Physica A 391:4704-4710

18. Nakamura T, Judd K, Mees AI, Small M (2006) A comparative study of information criteria for model selection. International Journal of Bifurcation and Chaos 16:21532175

19. Oda M, Iwashita K (2000) Study on couple stress and shear band development in granular media based on numerical simulation analyses. Int J Engng Sci 38:1713-1740 
20. Oda M, Kazama H (1998) Microstructure of shear bands and its relation to the mechanisms of dilatancy and failure of dense granular soils. Geotechnique 48:465-481

21. Peters JF, Walizer LE (2012) Patterned non-affine motion in granular media. Tech. Rep. ERDC/GSL TR-12-28, Geotechnical and Sturctures Laboratory, US Army Corps of Engineers

22. Ramesh MV (2009) Real-time wireless sensor network for landslide detection. In: Third International Conference on Sensor Technologies and Applications, 2009. SENSORCOMM'09., IEEE Conference Publications, pp 405-409

23. Rechenmacher A, Abedi S, Chupin O (2010) Evolution of force chains in shear bands in sands. Geotechnique 60(5):343

24. Rechenmacher AL, Abedi S (2011) Length scales for nonaffine deformation in localized, granular shear. In: Advances in Bifurcation and Degradation in Geomaterials, Springer Series in Geomechanics and Geoengineering, vol 11, Springer, pp 59-65

25. Rechenmacher AL, Abedi S, Chupin O (2011) Characterization of mesoscale instabilities in localized granular shear using digital image correlation. Acta Geotechnica 6:205-217

26. Rissanen J (1989) Stochastic complexity in statistical inquiry. World Scientific, Singapore

27. Small M (2005) Applied Nonlinear Time Series Analysis: Applications in Physics, Physiology and Finance volume 52 of Nonlinear Science Series A. World Scientific, Singapore

28. Small M, Judd K (1999) Detecting periodicity in experimental data using linear modeling techniques. Physical Review E 59(2):1379-1385

29. Suiker ASJ, Chang CS (2004) Modeling failure and deformation of an assembly of spheres with frictional contacts. Journal of Engineering Mechanics 130:283-293

30. Sutton MA, Orteau JJ, Schreier HW (2009) Image Correlation for Shape, Motion and Deformation Measurements: Basic Concepts, Theory and Applications. Springer: New York

31. Tanizawa T, Nakamura T (2011) Complex network from time series. In: 2011 International Symposium on Nonlinear Theory and its Applications, NOLTA2011, Kobe, Japan, pp 690-692

32. Tordesillas A (2007) Force chain buckling, unjamming transitions and shear banding in dense granular assemblies. Philosophical Magazine 87:4987-5016

33. Tordesillas A, Muthuswamy M (2009) On the modelling of confined buckling of force chains. Journal of Mechanics and Physics of Solids 57:706-727

34. Tordesillas A, Muthuswamy M, Walsh SDC (2008) Mesoscale measures of nonaffine deformation in dense granular assemblies. Journal of Engineering Mechanics - ASCE 134:1095-1113

35. Tordesillas A, Zhang J, Behringer RP (2009) Buckling force chains in dense granular assemblies: physical and numerical experiments. Geomechanics and Geoengineering $4: 3-16$

36. Tordesillas A, Walker DM, Lin Q (2010) Force cycles and force chains. Physical Review E 81:011,302

37. Tordesillas A, Shi J, Peters JF (2012) Isostaticity in cosserat continuum. Granular Matter 14:295-231

38. Utter B, Behringer RP (2008) Experimental measures of affin and nonaffine deformation in granular shear. Physical Review Letters 100:208,302

39. Walker DM, Tordesillas A (2012) Taxonomy of granular rheology from grain property networks. Physical Review E 85:011,304 
40. Walker DM, Tordesillas A, Nakamura T, Tanizawa T (2013) Directed network topology from grain stress dynamics. Physical Review E 87:032,203

41. Werner-Allen G, Lorincz K, Ruiz M, Marcillo O, Johnson J, Lees J, Welsh M (2006)

Deploying a wireless sensor network on an active volcano. Internet Computing, IEEE 10:18-25

42. Whittle P (1971) Optimization Under Constraints. Wiley, Chichester 


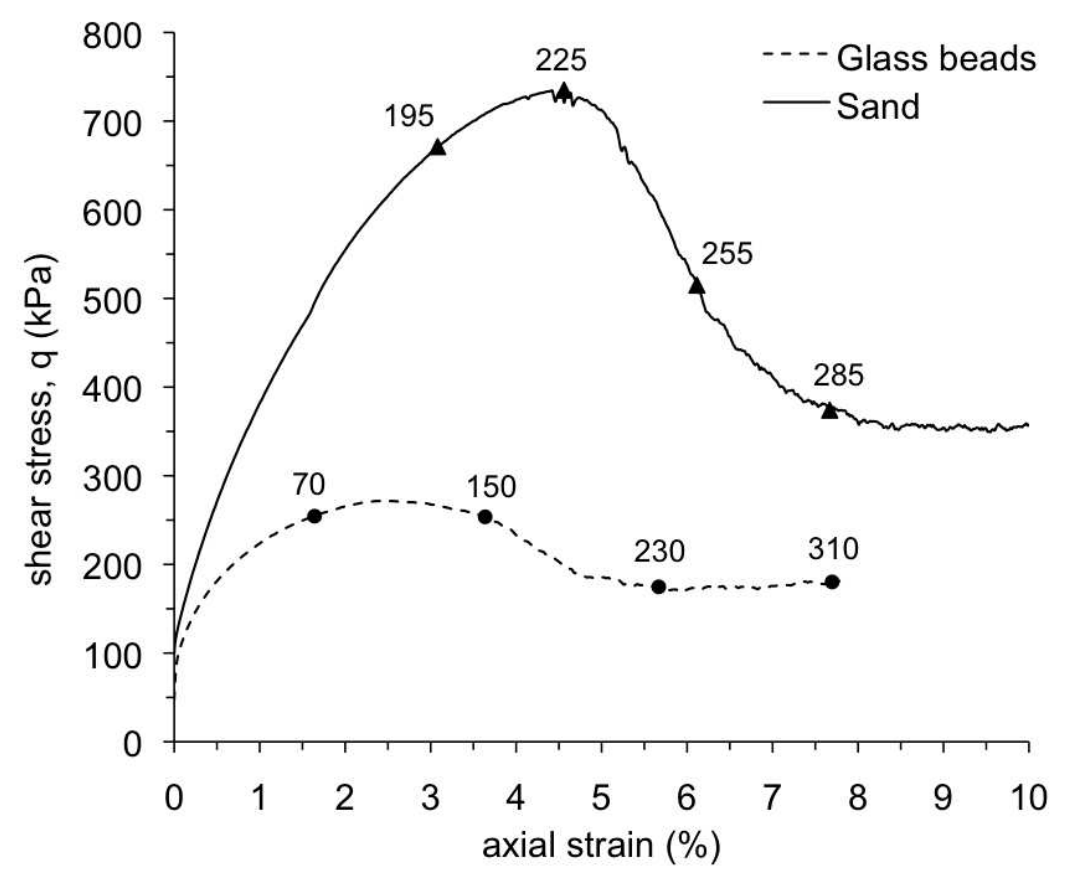

Fig. 1 Stress-strain evolution and the endpoints of the DIC measurement increments for the glass beads and MC sand experiments. 
Strain increment:i70-78 Strain increment:1142-150
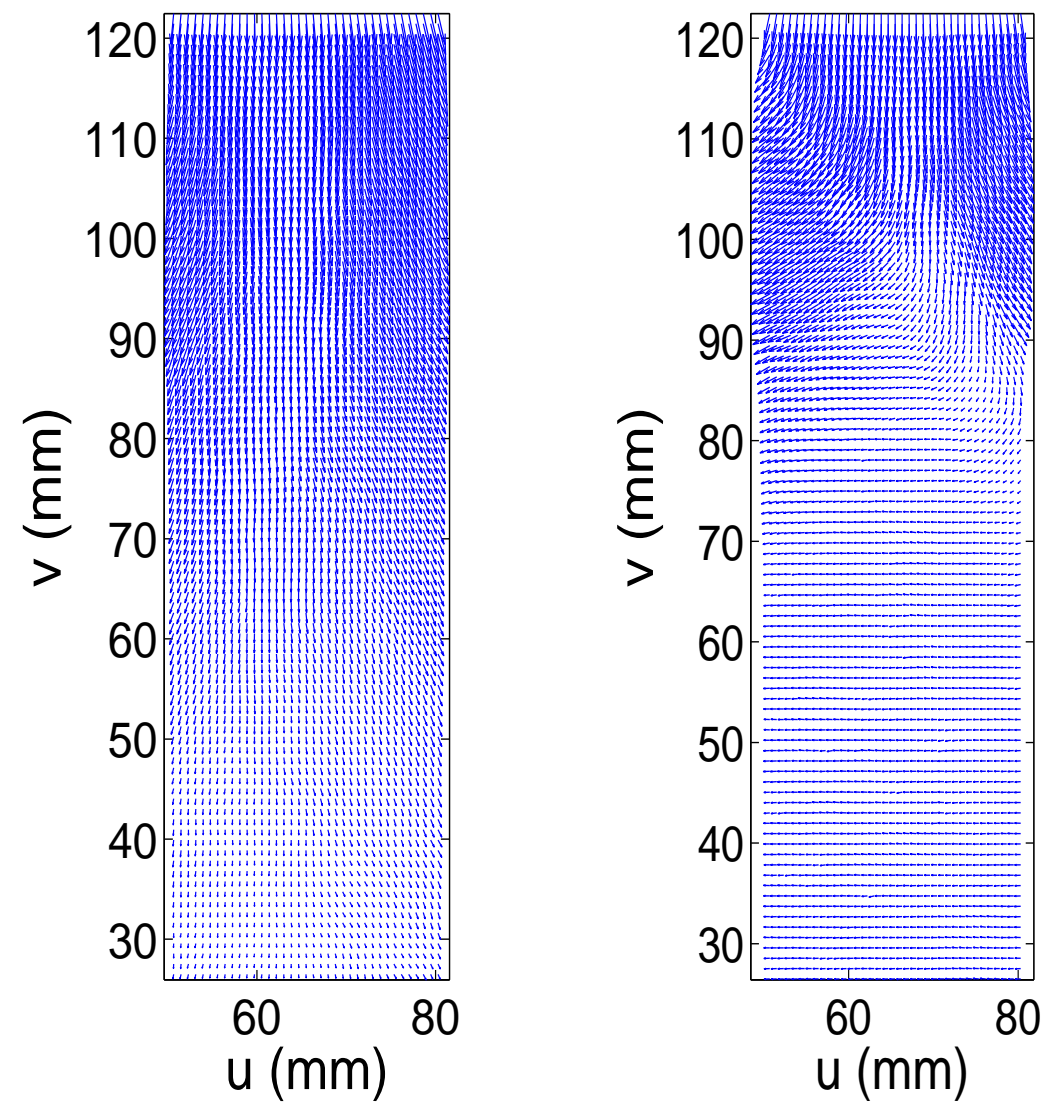

Strain increment:i222-230 Strain increment:i302-310
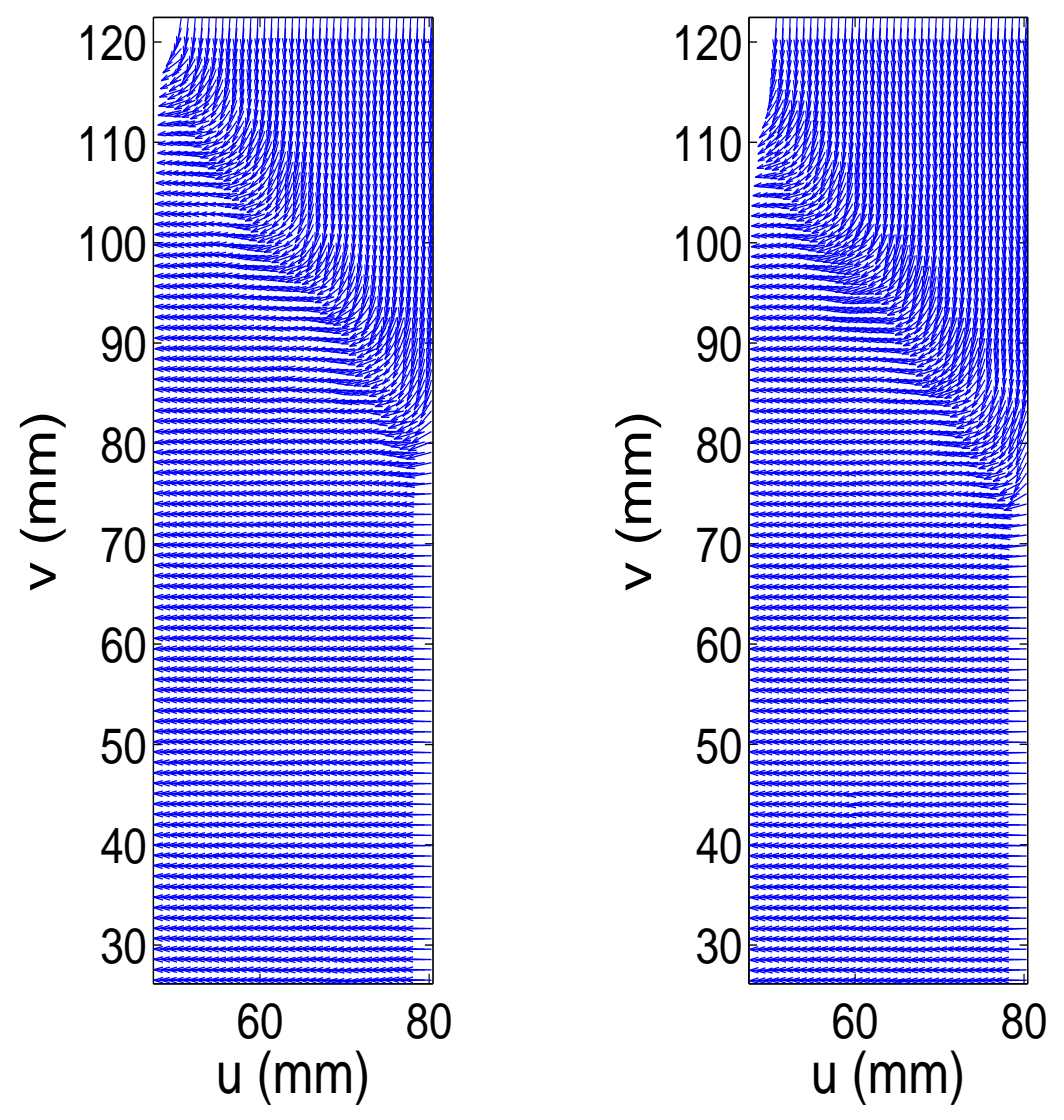
Strain increment:195-198 Strain increment:222-225
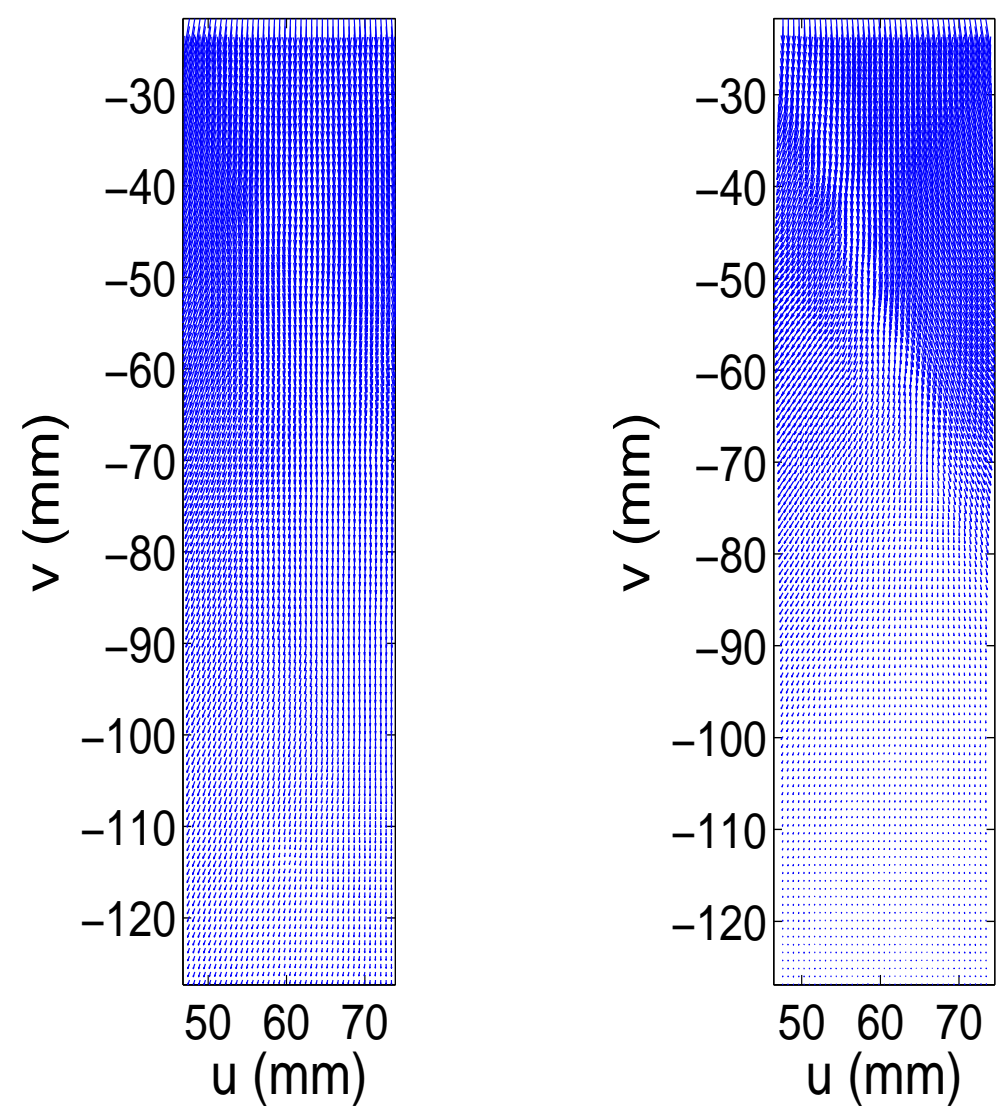

Strain increment:252-255 Strain increment:282-285
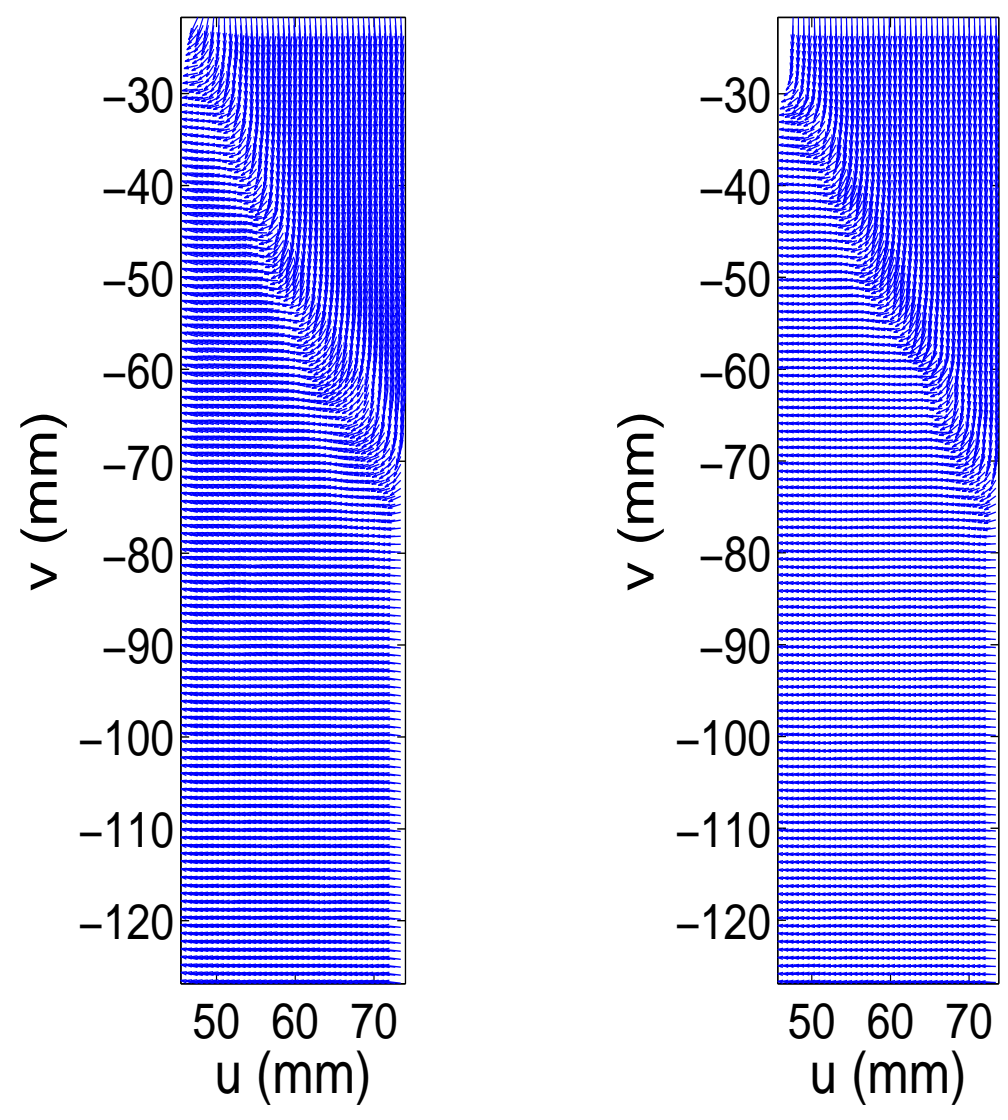


\section{Glass beads observation sites}

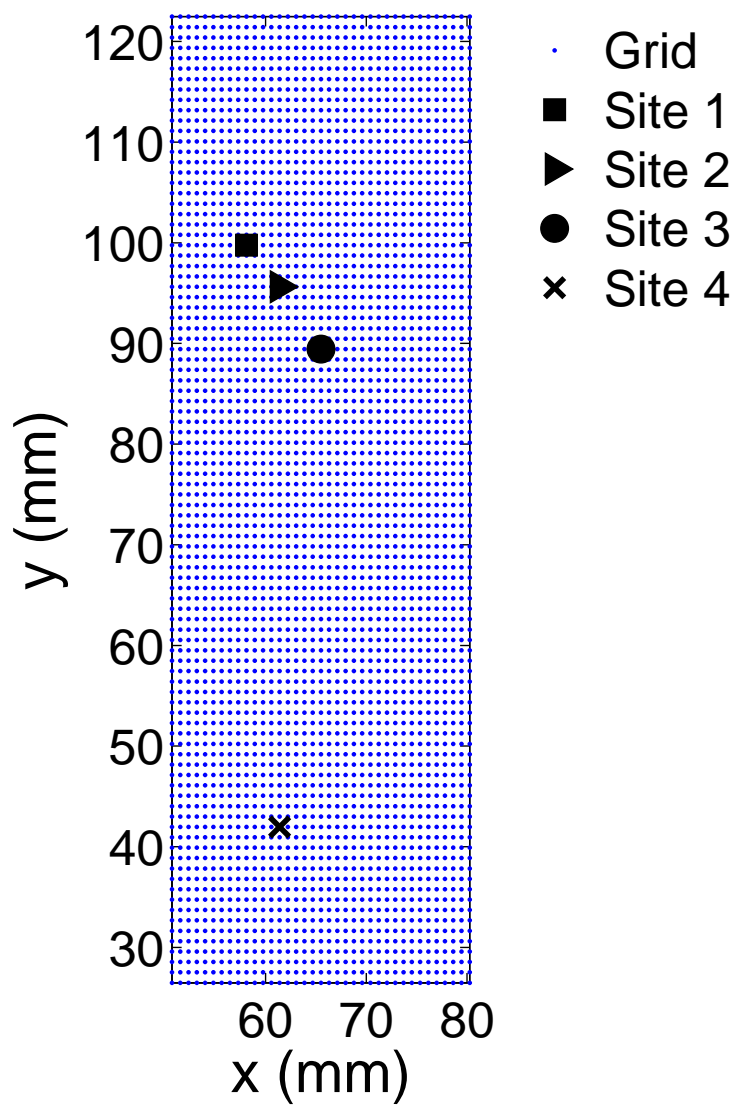

Fig. 4 Site locations for three sensors in the shear band and one outside for the glass beads sample. These sites reference traces shown in Fig. 5. 

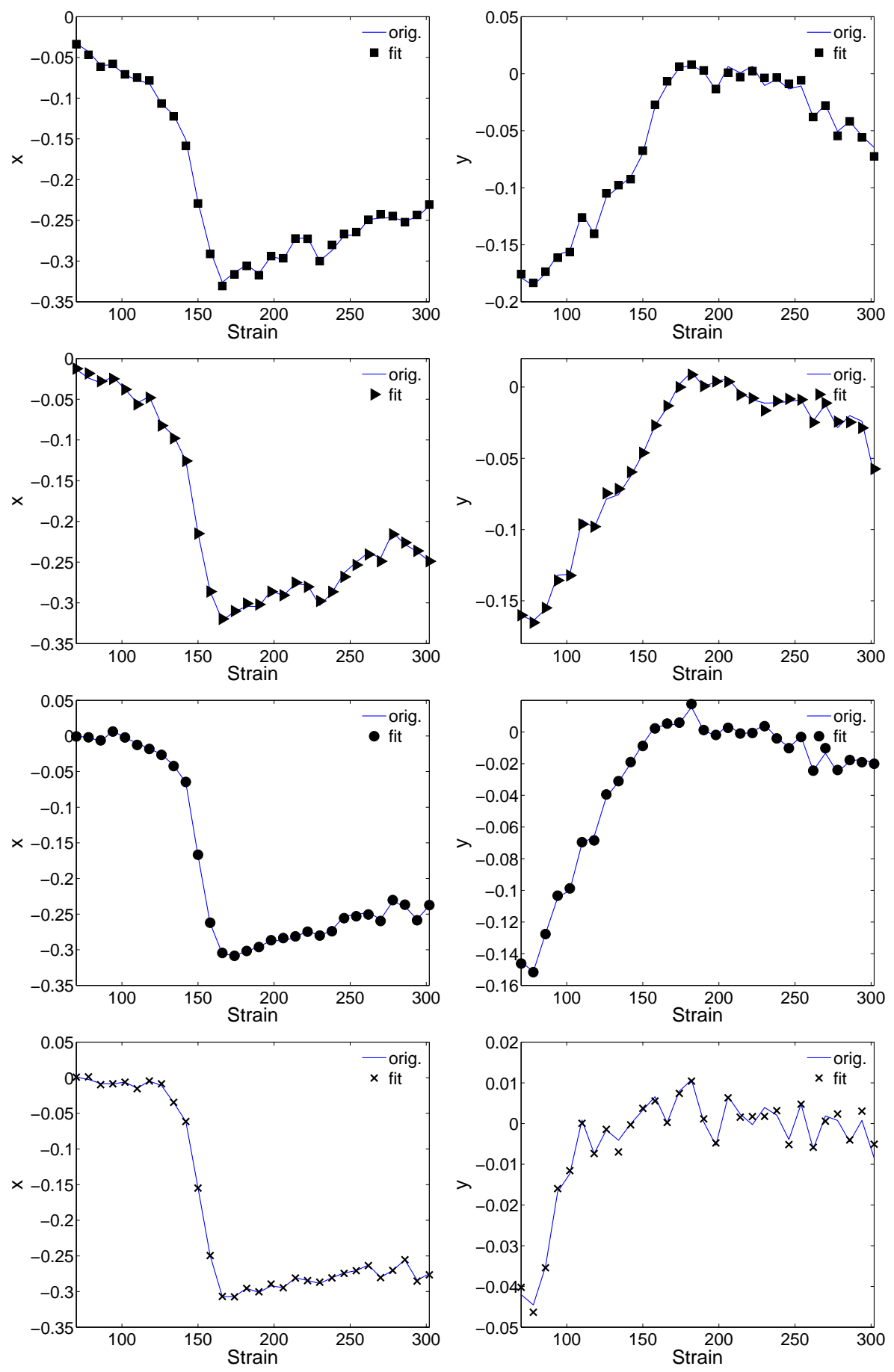

Fig. 5 The data (solid line) and the fitted SLR predictions (symbols) for the $x$ - (left) and $y$ - (right) components of the sensor sites shown in Fig. 4. In all fits, the training errors are of zero mean and a standard deviation an order of magnitude less than the data range, with the poorest predictions occurring for sensor sites in the eventual shear band location. 


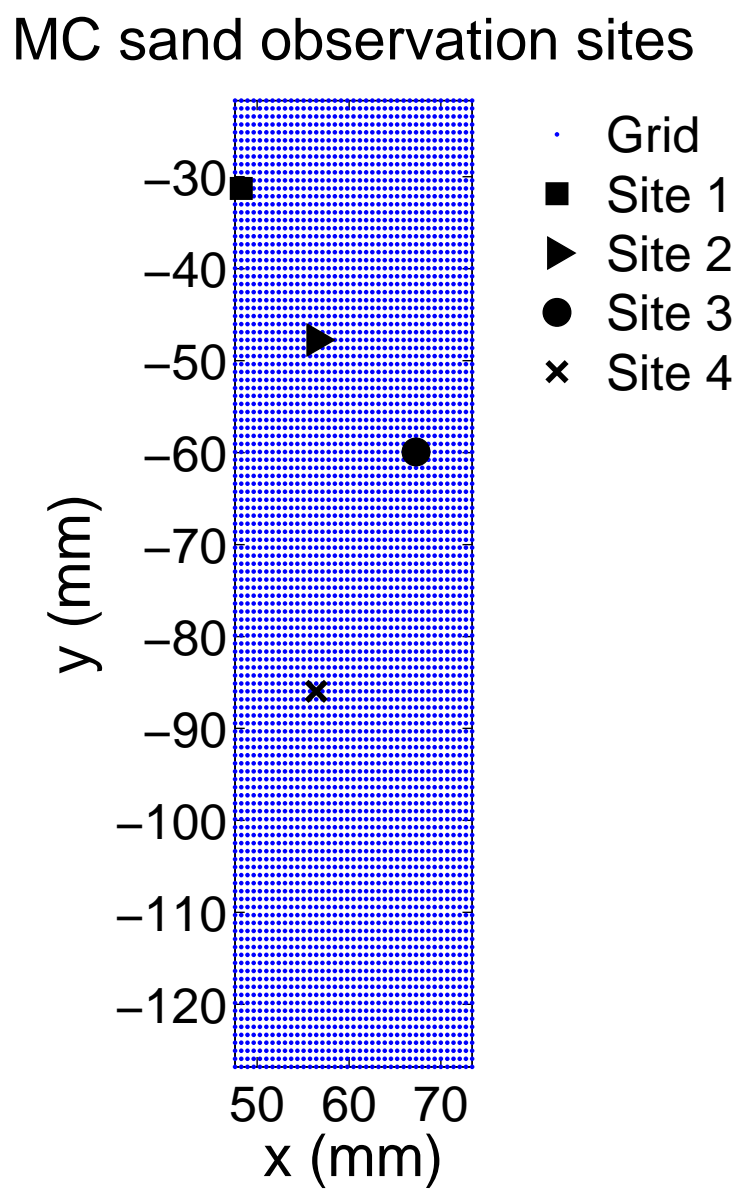

Fig. 6 Site locations for three sensors in the shear band and one outside for the MC sand sample. These sites reference the traces shown in Fig. 7. 

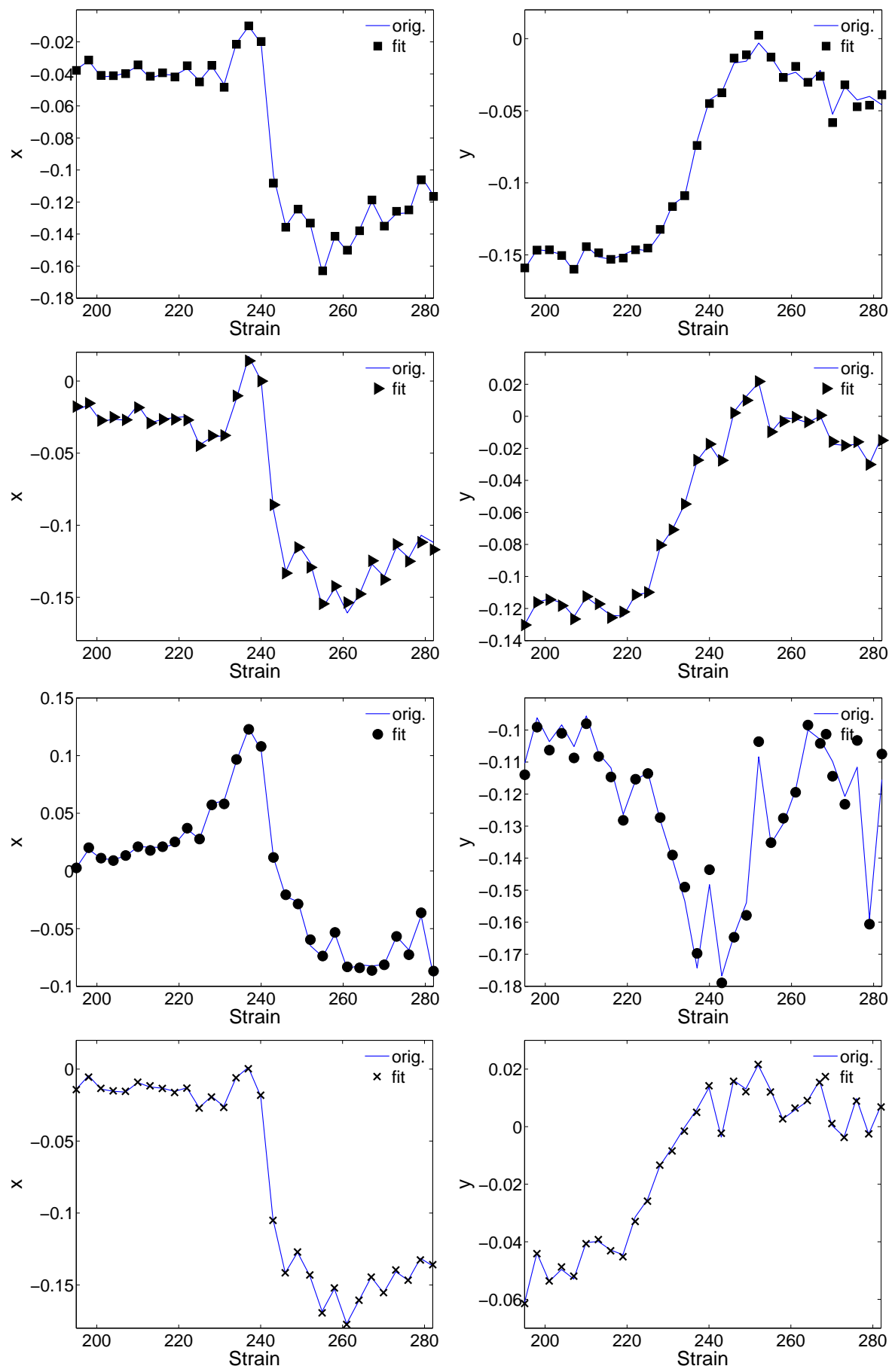

Fig. 7 The data (solid line) and the fitted SLR predictions (symbols) for the $x$ - (left) and $y$ - (right) components of the sensor sites shown in Fig. 6. In all fits, the training errors are of zero mean and a standard deviation an order of magnitude less than the data range, with the poorest predictions occurring for sensor sites in the eventual shear band location. 

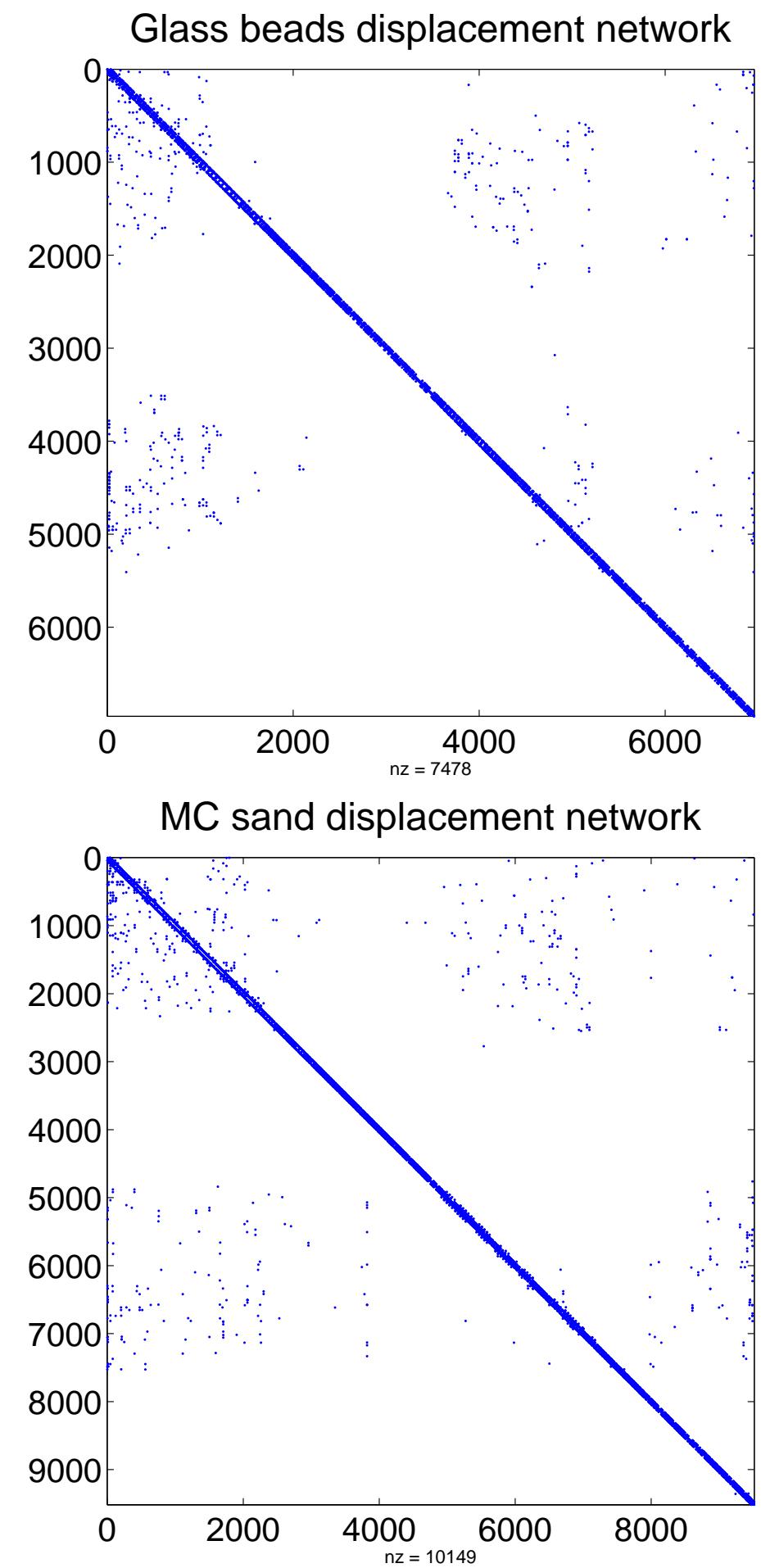

Fig. 8 Adjacency matrix of the directed networks: glass beads SLRs and MC sand SLRs. The filled dots show the "nz" nonzero entries in each adjacency matrix. Row sums give the in-degree values whereas column sums give the out-degree. The first 3478 (4758) rows and columns represent the $x$-coordinate information and the remaining rows and columns represent the $y$-coordinate information of the glass bead (MC sand) SLRs. Observe most SLRs use local information of the same type (i.e., dominant diagonal); however there are some sites whose SLR require mixed $x$ - and $y$-displacement information. 

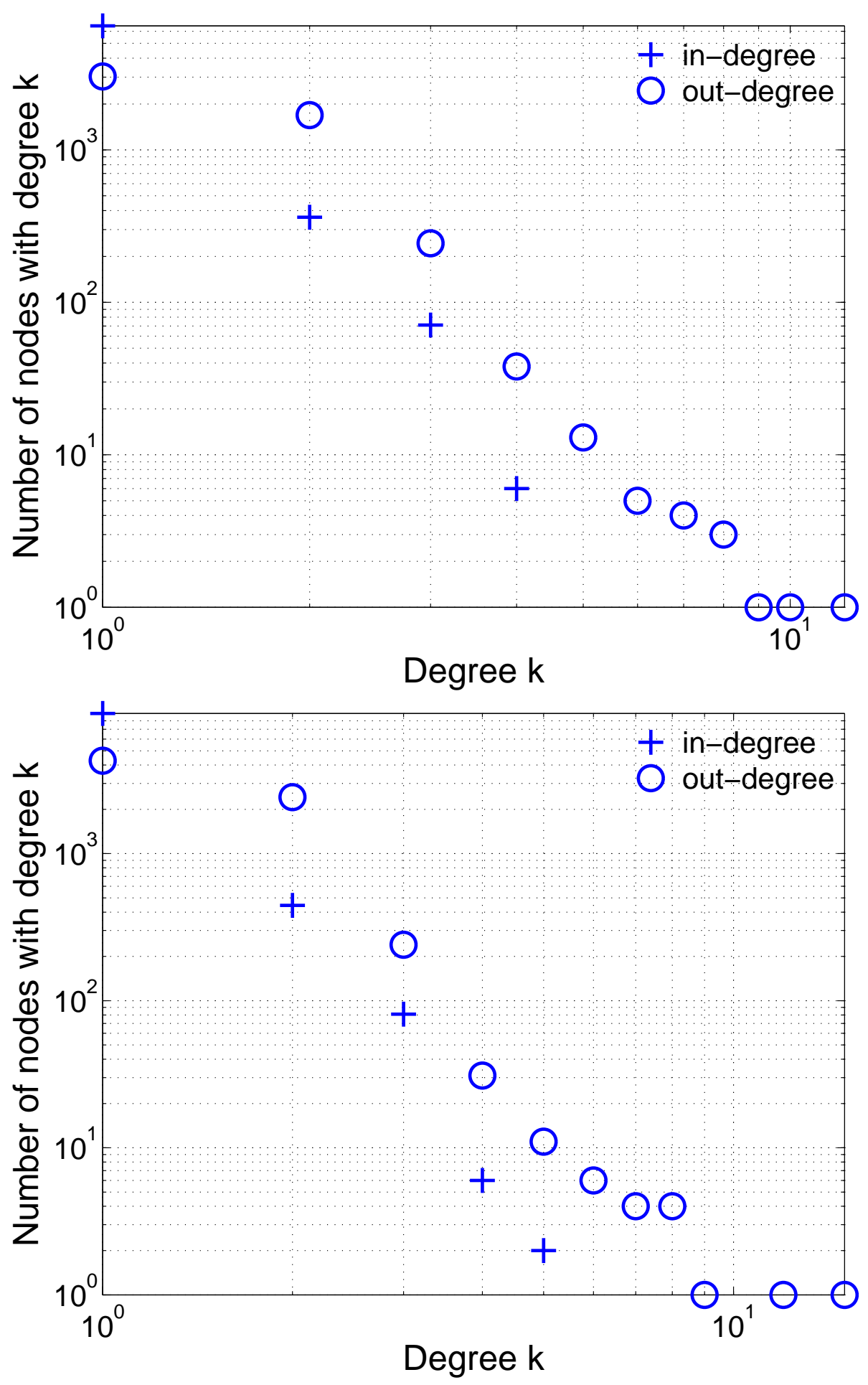

Fig. 9 Empirical in-degree and out-degree distributions plotted on a log-log scale: glass beads SLR (upper) and MC sand SLR (lower). 

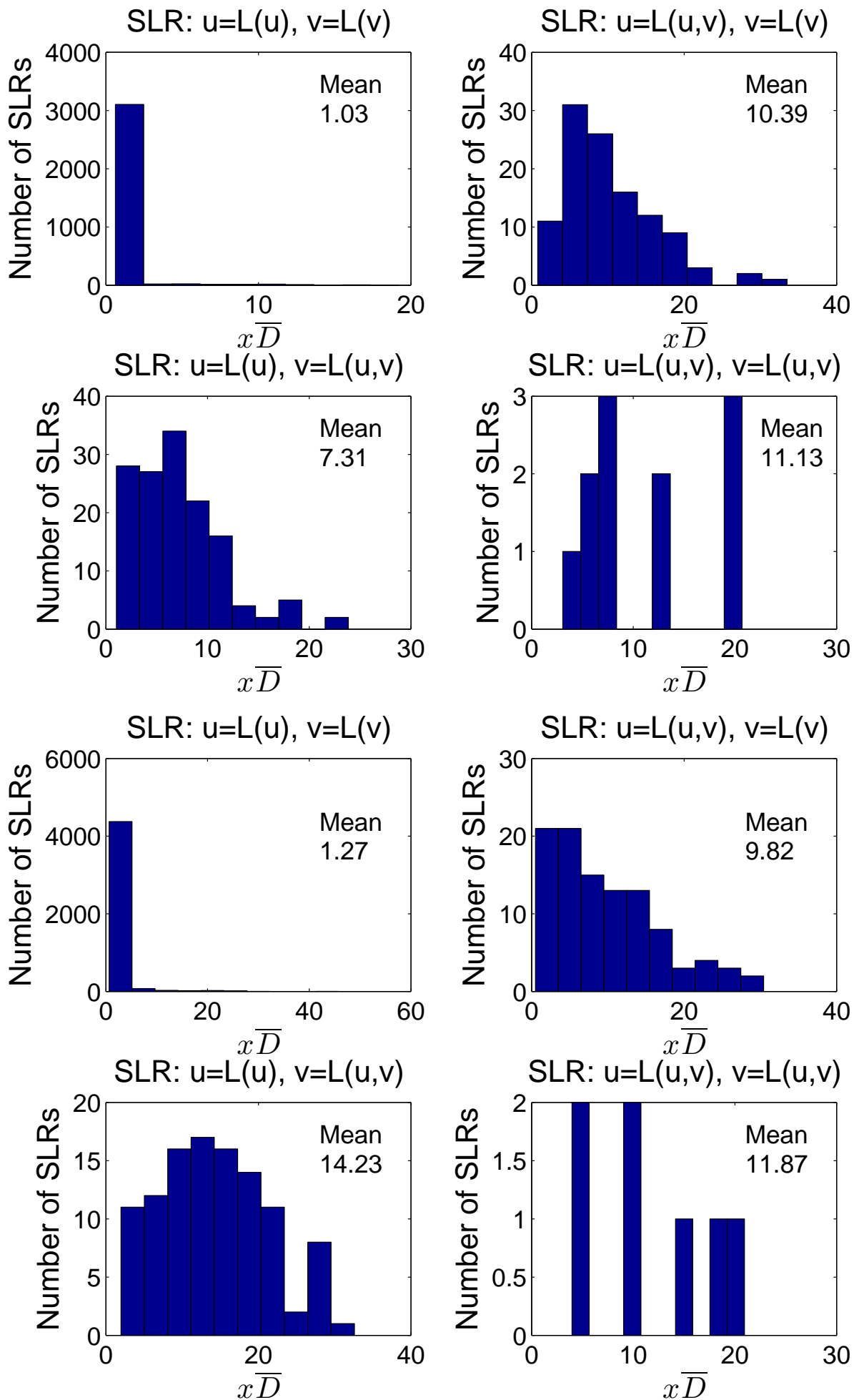

Fig. 10 Average distance from a site to its selected SLR sensors with respect to $\bar{D}$ — the size of an observation site (Euclidean distance of grid diagonal). Upper (four): Glass beads SLR. Lower (four): MC sand SLR. SLR structure as indicated. The extent of mixed and nonlocal SLR sites are typically within the order of $(7-15) \bar{D}$ sites away for both samples. 


\section{Glass beads displacement network}

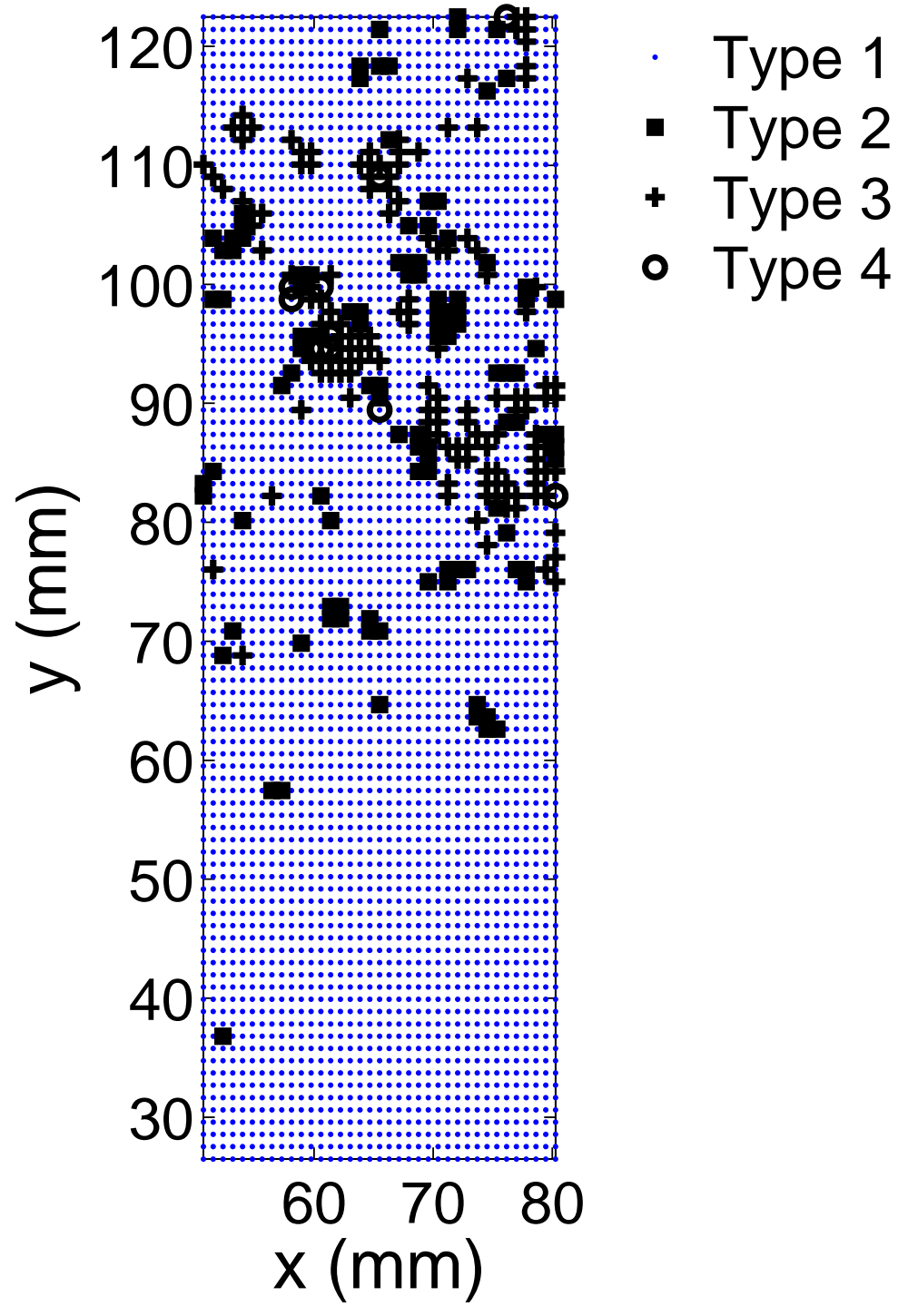

Fig. 11 Visualizing the broad features of the glass beads SLR models. Types represent the following: sites whose SLRs can be summarized as Type $1-u=L(u), v=L(v)$, Type $2-u=L(u, v), v=L(v)$, Type 3 $-u=L(u), v=L(u, v)$ and Type $4-u=L(u, v), v=L(u, v)$. Observe that SLR's with mixed and nonlocal information appear to delineate the shear band. 


\section{MC sand displacement network}

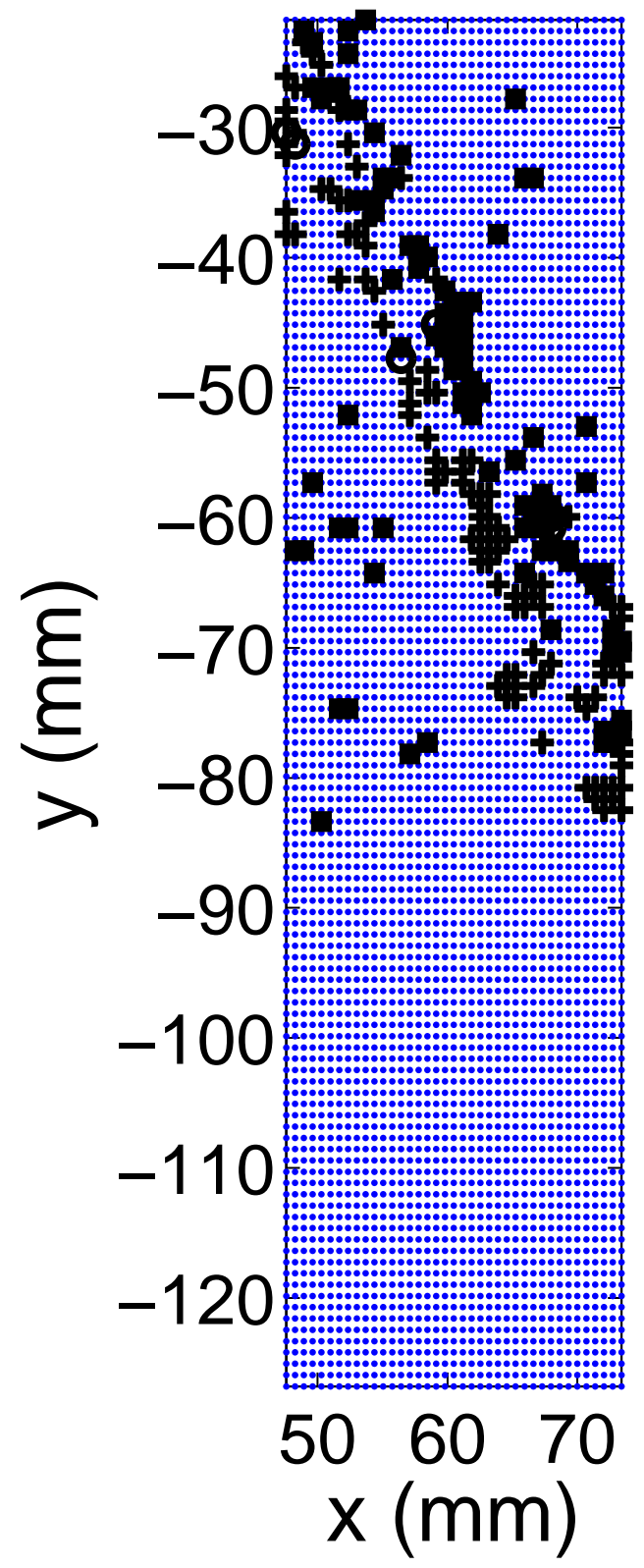

Fig. 12 Visualizing the broad features of the MC sand SLR models. Types represent the following: sites whose SLRs can be summarized as Type $1-u=L(u), v=L(v)$, Type $2-u=L(u, v), v=L(v)$, Type 3 $-u=L(u), v=L(u, v)$ and Type $4-u=L(u, v), v=L(u, v)$. Observe that SLR's with mixed and nonlocal information appear to delineate the shear band. 


\section{University Library}

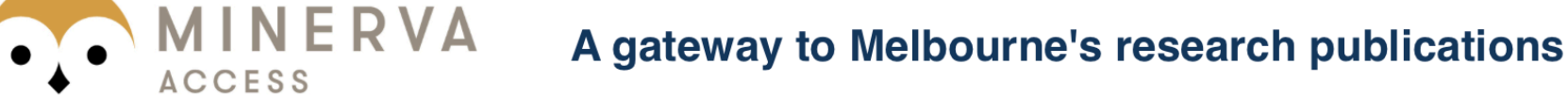

Minerva Access is the Institutional Repository of The University of Melbourne

Author/s:

Walker, DM;Tordesillas, A;Rechenmacher, AL

Title:

Transmission of kinematic information in dense granular systems: local and nonlocal network sensing

Date:

2013-10-01

Citation:

Walker, D. M., Tordesillas, A. \& Rechenmacher, A. L. (2013). Transmission of kinematic information in dense granular systems: local and nonlocal network sensing. ACTA GEOTECHNICA, 8 (5), pp.547-560. https://doi.org/10.1007/s11440-013-0266-z.

Persistent Link:

http://hdl.handle.net/11343/282574 